\title{
Development of Automatic Magnification Calibration Function for Scanning Transmission Electron Microscope
}

\author{
Hiromi Inada*, D. Terauchi*, M. Ozawa**, R. Tsuneta***, H. Tanaka*, M. Konno**, S. Watanabe*, \\ S. Aizawa*, K. Nakamura*** and A. Takane* \\ * Hitachi High-Technologies, 882, Ichige, Hitachinaka, Ibaraki 312-8504 Japan \\ ** Hitachi Science Systems, 1040 Ichige, Hitachinaka, Ibaraki 312-0033 Japan \\ *** Central Research Laboratory, 1-280, Higashi-Koigakubo, Kokubunji, Tokyo 185-8601 Japan
}

Scanning transmission electron microscopes (STEM) are ideally suited to address the growing demand for the metrology of features which range from a few nanometers to tens of nanometers in size. The metrology must be carried out with a high degree of accuracy and repeatability. For metrology with scanning electron microscopes (SEM) or critical dimension SEMs (CD-SEM) the magnification is calibrated by using a Hitachi's standard micro scale specimen whose pitch is guaranteed by JQA (Japan Quality Assurance Organization). With a pitch of 240nm these micro scale specimens can be used to calibrate the magnifications used for many line-width applications. However, the metrology of smaller features such as gate oxides or ONO layers requires magnifications from ten to one hundred times higher than can be calibrated with the micro scale specimen. Calibrating the magnifications used to measure nanometer scale features has been difficult and researchers are still in need of the appropriate calibration specimen.

A HD-2300 STEM (Hitachi High Technologies) guarantees a $0.2 \mathrm{~nm}$ spot size [1]. This STEM is equipped with three detectors, for secondary electron (SE), dark field (DF) and bright field (BF) STEM imaging. Elemental mapping can be performed using EDX or by using the ELV-2000 Hitachi EELS mapping system. The HD-2300 can observe and analyze samples over a wide range of magnifications. Low magnifications $(500-50 \mathrm{kx})$ are useful for imaging the construction of transistors and their interconnects, and can be calibrated using the standard micro scale specimens. High magnification images $(>1000 \mathrm{kx})$ are required for measuring gate oxides, and for observing lattice fringes. This report describes an automatic magnification calibration function for the high magnification range required to accurately measure features from a few to tens of $\mathrm{nm}$ in size.

The automated magnification calibration function requires only that the user obtain a lattice image from $\mathrm{Si}$ or from some other material with known lattice constants. By relying on lattice images, no special calibration sample is required, and the operator can in many cases use the sample's substrate. The function is carried out by processing the image using the Fast Fourier Transform (FFT) method, which makes calibration fast and easy on the PC which controls the HD2300. The FFT method averages lattice information from the whole image and distinguishes the desired pitch. Figure 2. shows a brief flow chart of this function, and the process is described as follows:

First lattice fringe images of an Si crystal are captured at selected magnification (1). Next the images are moved into the magnification calibration GUI window of the HD-2300 (2). The FFT calculation is then carried out automatically to measure the magnification error of the equipment (3). Finally, the result of this error calculation is fed back to the HD-2300 controller to compensate for the magnification error (4).

By using this automatic magnification calibration function, the magnification error is held to less than $1 \%$ and 3 sigma repeatability is $1.9 \%$ at a magnification of $1,500,000$ times. High accuracy metrology for semiconductor and nanotechnology features ranging from a few $\mathrm{nm}$ to tens of $\mathrm{nm}$ in 
size is made possible by using this function. Results of magnification calibration in the middle to high range and several applications are reported here.

\section{References}

[1] H. Inada et al., Proceedings of 8th APEM. (2004) 60.

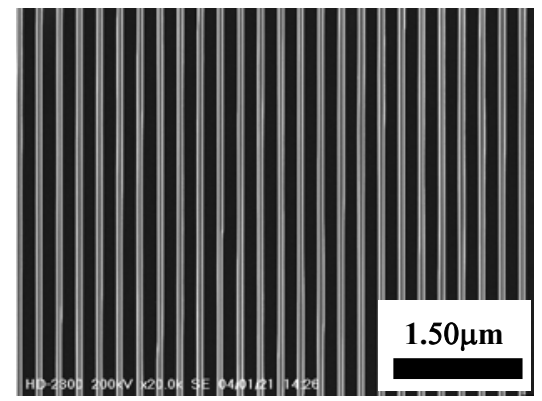

FIG. 1. An image of $240 \mathrm{~nm}$ spacing micro scale specimen which calibrates magnification for SEM.

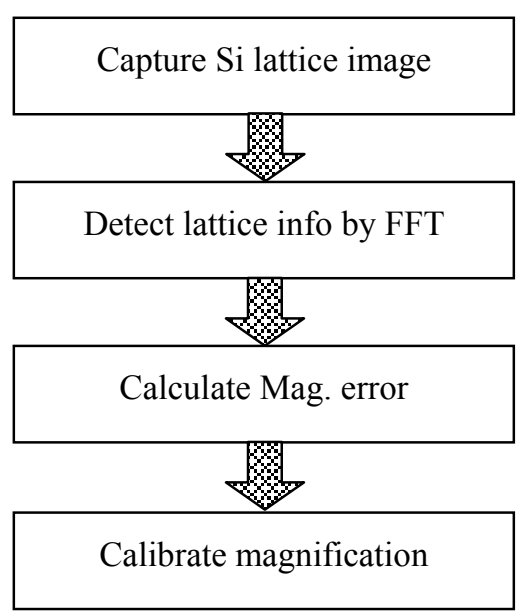

FIG. 2. A brief flow chart of automated magnification calibration function.
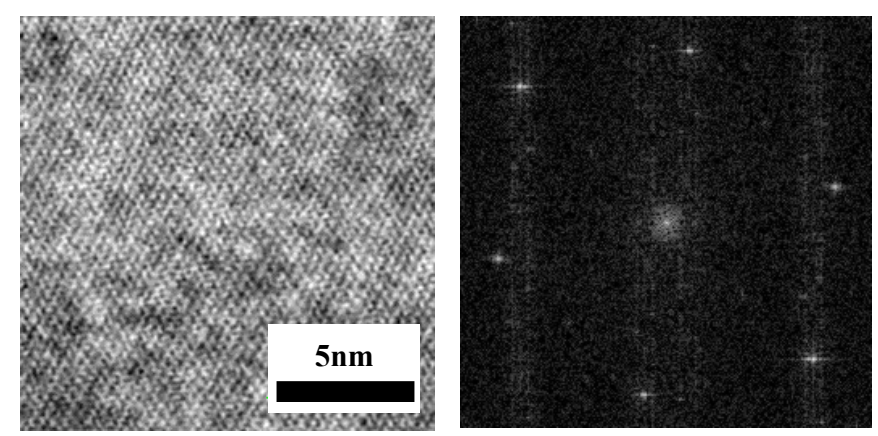

FIG. 3. Image of Si lattice fringe taken by HD-2300 (left) and its FFT calculated image (right). The function detects bright spot position which corresponds to lattice information. There are three lattice space in the image taken, two $0.313 \mathrm{~nm} \mathrm{Si}(111)$ and one $0.272 \mathrm{~nm} \mathrm{Si}(200)$. 